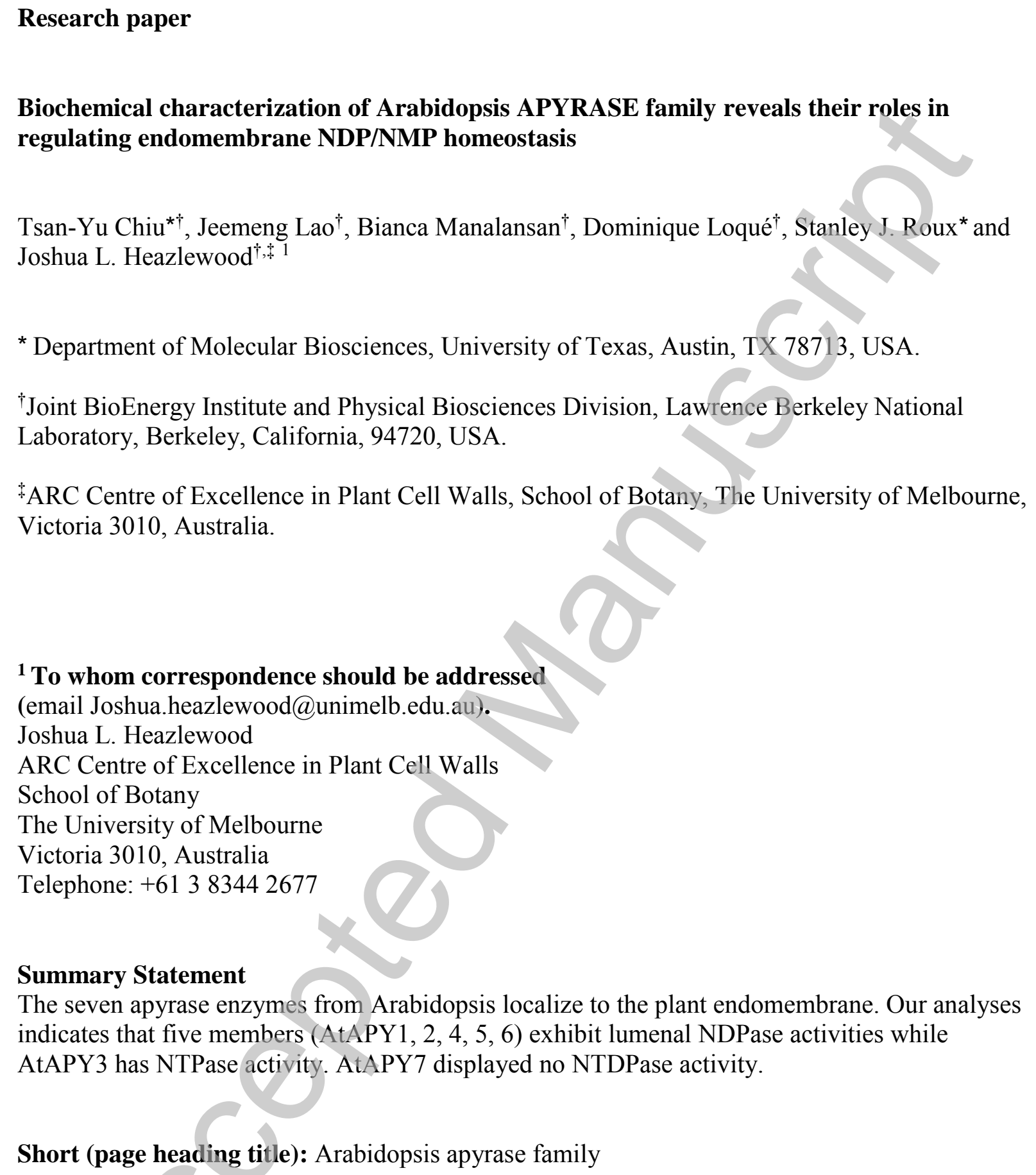

\section{Summary Statement}

The seven apyrase enzymes from Arabidopsis localize to the plant endomembrane. Our analyses indicates that five members (AtAPY1, 2, 4, 5, 6) exhibit lumenal NDPase activities while AtAPY3 has NTPase activity. AtAPY7 displayed no NTDPase activity.

Short (page heading title): Arabidopsis apyrase family 


\title{
41 Abstract
}

\begin{abstract}
Plant apyrases are nucleoside triphosphate diphosphohydolases and have been implicated in an array of functions within the plant including the regulation of extracellular ATP. Arabidopsis encodes a family of seven membrane bound apyrases (AtAPY1 to 7) comprised of three distinct clades all of which contain the five conserved apyrase domains. With the exception of AtAPY1 and AtAPY2, the biochemical and the subcellular characterization of the other members are currently unavailable. In this research, we have shown all seven Arabidopsis apyrases localize to internal membranes comprising the cis-Golgi, ER and endosome, indicating an endo-apyrase classification for the entire family. In addition all members, with the exception of AtAPY7, can function as endo-apyrases by complementing a yeast double mutant ( $\Delta y n d 1 \Delta$ gda1) which lacks apyrase activity. Interestingly to note that complementation of the mutant yeast using well characterized human apyrases could only be accomplished by using a functional ER endoapyrase (NTPDase6), but not the ecto-apyrase (NTPDase1). Furthermore, the substrate specificity analysis for the Arabidopsis apyrases AtAPY1 to 6 indicated that each member has a distinct set of preferred substrates covering various NDPs and NTPs. Combining the biochemical analysis and subcellular localization of the Arabidopsis apyrases family, the data suggests that their possible roles in regulating endomembrane NDP/NMP homeostasis.
\end{abstract}

\section{Keywords \\ Arabidopsis apyrase, human, yeast, endomembrane}

Abbreviations: NTPDase, nucleoside triphosphate diphosphohydolases; ACR, apyrase conserved regions; ATPases, adenosine triphosphatases; ER, endoplasmic reticulum; dKO, double knock-out 


\section{Introduction}

The apyrase class of enzymes (EC 3.6.1.5) are nucleoside triphosphate diphosphohydolases (NTPDases) that belong to the GDA1_CD39 nucleoside phosphatase superfamily and contain five apyrase conserved regions (ACRs). They are active against both nucleotide tri- and diphosphates (NTP, NDP), converting them to nucleotide monophosphates (NMP). Apyrases have been identified in an array of species, including plants, mammals, insects, fungi and bacteria [1]. The NTPDase activity requires divalent cations $\left(\mathrm{Mg}^{2+}, \mathrm{Ca}^{2+}\right)$ and are distinct from the adenosine triphosphatases (ATPases) due to their broader substrate activities and insensitivities to F-type, P-type, and V-type ATPase inhibitors [2].

In mammals, apyrases were initially characterized as having cell surface ATPase activity (ectoapyrase). The human apyrases are the most extensively characterized family and comprise cell surface localized ecto-apyrases (NTPDases 1, 2, 3 and 8) and endo-apyrases which are associated with the endoplasmic reticulum (ER), Golgi and intracellular vesicles (NTPDase 4, 5, $6,7)[1]$. The plasma membrane localized apyrases are mainly involved in the regulation of extracellular ATP to prevent desensitization of purine receptors [3]. In contrast, the intracellular localized ER/Golgi human endo-apyrases are involved in the conversion of NDP to NMP to both drive lumenal glycosylation reactions and produce co-substrates (NMP) for the membrane localized nucleotide sugar antiporters [4]. Saccharomyces cerevisiae (yeast) encodes two apyrases, namely GDA1 and YND1 [5, 6]. The yeast GDA1 protein is an NDPase with preferential activity against GDP [7]. YND1 has a broader substrate specificity and can readily hydrolyze both NDPs and NTPs, although with a preference for GDP [6]. The functions of these two yeast apyrases are somewhat redundant as YND1 can partially complement glycosylation defect phenotypes when expressed in the $\Delta g d a 1$ background. Interestingly, yeast cells ( $\Delta y$ nd1 $\Delta g d a 1)$ lacking both apyrases are still viable [6].

In plants, the involvement of extracellular ATP as a potential signaling molecule has been proposed for a number of years [8]. A number of studies have demonstrated that plant cells release significant quantities of ATP into their extracellular matrix when they are mechanically stimulated [9], wounded [10], during growth [11] and during stomatal opening [12]. Recently, with the characterization of a plasma membrane localized ATP receptor kinase [13] a role for plant apyrases in the regulation of extracellular ATP has been strengthened. In the reference plant Arabidopsis thaliana, a total of seven NTPDases have been identified based on the presence of the ACRs [14]. Among the seven members, APYRASE 1 (AtAPY1 At3g04080) and APYRASE 2 (AtAPY2: At5g18280) have been the most extensively investigated. Both AtAPY1 and AtAPY2 have been shown to play numerous physiological roles in pollen development, vegetative growth and stomata opening/closure $[12,15,16]$. Collectively, these responses were attributed to defects in ecto-nucleotide signaling responses. However, recently both AtAPY1 and AtAPY2 have been identified in plant Golgi proteomes [17] and their localizations confirmed by fluorescent protein tagging $[18,19]$. In addition, knocking out either AtAPY1 or AtAPY2 affects latent lumenal UDPase/GDPase activity in microsomal preparations from Arabidopsis which resulted in a minor change to the galactose content of their cell walls [18]. Furthermore, the conditional suppression of AtAPY1 in the atapy2 background resulted in structural changes to the cell wall [20]. These data provide strong evidence to support of the hypothesis that AtAPY1 and AtAPY2 functions as plant endo-apyrases and are necessary for lumenal glycosylation. However, this functionally defined role as an endo-apyrase would not necessarily preclude a role 
as regulators of ecto-ATP/ADP concentration via secretary mechanism, as has been recently argued [14] based on data showing that immunochemical [16] and genetic [20] suppression of AtAPY1 and AtAPY2 results in an increase in extracellular ATP.

Aside from AtAPY1 and AtAPY2, a further five apyrase members are encoded by Arabidopsis (AtAPY3 to 7), although their biochemical and physiological functions remain elusive. Some initial characterization of AtAPY6 and AtAPY7 has been undertaken, with double knock-out plants (atapy6atapy7) resulting in late anther dehiscence, exine deformation and low male fertility [21]. These structural changes to the pollen cell wall in combination with an internal localization for AtAPY6 tagged lines further support roles as endo-apyrases involved in polysaccharide biosynthesis [21]. Consequently, in an effort to resolve the functional roles of the Arabidopsis apyrase family, we sought to systematically investigate their subcellular localizations and determine their substrate specificities and relate these findings to functional roles in the context of the well characterized apyrase family members from humans. 


\section{Experimental}

\section{Cloning procedures for heterologous protein expression}

The Arabidopsis apyrase family members AtAPY3 (At1g14240), AtAPY4 (At1g14230), AtAPY5 (At1g14250), AtAPY6 (At2g02970) and AtAPY7 (At4g19180) were cloned from a mixed organ Arabidopsis cDNA library using primers designed based on sequences in The Arabidopsis Information Resource (TAIR) [22] (Table S1). PCR products were recombined into pDONR ${ }^{\mathrm{TM}} /$ Zeo by BP reaction (Life Technologies) and verified by sequencing. The genes AtAPY1 (At3g04080) and AtAPY2 (At5g18280) were previously cloned using a similar approach [17]. For transient subcellular localizations, the AtAPY1 to $7 \mathrm{pDONR}^{\mathrm{TM}} / \mathrm{Zeo}$ constructs were recombined into the N-terminal YFP and C-terminal YFP Gateway ${ }^{\circledR}$ compatible pBullet vectors [23] by LR reactions (Life Technologies). The human apyrase cDNA sequences were obtained from the Mammalian Gene Collection [24] and comprised ENTPD1 (BC047664.1), ENTPD6 (BC025980.2) and ENTPD7 (BC122857.1). These sequences were codon optimized for yeast expression (Figure S1), synthesized (GenScript), recombined into the $\mathrm{pDONR}{ }^{\mathrm{TM}} / \mathrm{Zeo}^{\mathrm{N}}$ vector by $\mathrm{BP}$ reaction (Life Technologies) and verified by sequencing. The AtAPY7 sequence was codon optimized (Figure S1) and synthesized (GenScript) for yeast expression. For yeast complementation assays, the $\mathrm{pDONR}^{\mathrm{TM}} / \mathrm{Zeo}$ constructs were recombined into a pDR-Leu Gateway® yeast expression vector [17].

\section{Chromosomal deletion the GDA1 locus from Saccharomyces cerevisiae}

The chromosomal GDA1 locus (YEL042W) was replaced with URA3 (orotidine-5'-phosphate) by homologous recombination as previously described [25]. Genomic DNA was extracted from the Saccharomyces cerevisiae wild type strain BY4741 (MATa, his341, leu240, met1540, ura3 40 ) using YeaStar ${ }^{\mathrm{TM}}$ Genomic DNA Kit (Zymoresearch) and used as template. The yeast GDA1 gene was cloned by PCR using the pGDA1 primers and inserted into pENTR ${ }^{\mathrm{TM}} / \mathrm{D}-$ TOPO ${ }^{\circledR}$ (Life Technologies) to be used as the templates to create the knockout cassette border sequence. A further round of PCR was undertaken using the pGDA1-R primers to create a BamHI site used to replace GDA with URA3. The resultant product was cloned into pENTR ${ }^{\mathrm{TM}} / \mathrm{D}-\mathrm{TOPO}{ }^{\circledR}$. The URA3 gene was amplified by PCR from the vector pRS416-GPD [26] and restriction sites BamHI and MscI were added by PCR using the URA3 primers. The URA3 PCR product was digested with BamHI and MscI and ligated into the pENTR/D-TOPOGDA backbone to create the knockout cassette pGDA-URA3-tGDA. To knockout the chromosomal GDA1 gene, the Saccharomyces cerevisiae strain $\triangle y n d 1$ (MATa, his341, leu2 40 ,

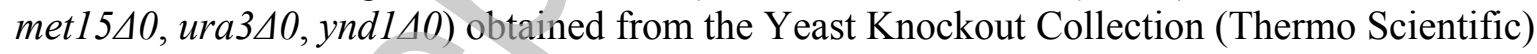
was transformed with the linearized vector pGDA-URA3-tGDA using Frozen-EZ Yeast Transformation II Kit ${ }^{\mathrm{TM}}$ (Zymoresearch). The transformants were selected on solid medium containing Yeast Nitrogen Base (YNB) without amino acids (Becton, Dickinson and Company) supplemented with $2 \%$ (w/v) glucose and 1X CSM-Ura (Sunrise Science Products). Genomic DNA was isolated from candidate transformants using YeaStar ${ }^{\mathrm{TM}}$ Genomic DNA Kit (Zymoresearch). The integrity of the GDA1 locus was examined by PCR using the following primer sets: left border using primers GDA-L; middle using primers GDA-M and right border using primers GDA3-R. The presence of the inserted URA3 sequence was examined by PCR using the primers URA3-ORF. Primers are detailed in Table S1.

\section{Yeast transformation and complementation assay}


The $\Delta g d a 1 \Delta y n d 1 \mathrm{dKO}$ yeast strain was transformed with the various plasmids using the EZYEAST $^{\mathrm{TM}}$ transformation kit (MP Biomedicals) and selected on solid media containing Yeast Nitrogen Base (YNB) without amino acids (Becton, Dickinson and Company) supplemented with $2 \%(\mathrm{w} / \mathrm{v})$ glucose and 1X CSM-Leu-Ura (Sunrise Science Products). Complementation was assessed by growing single colonies overnight at $30^{\circ} \mathrm{C}$ in liquid media as described above. Liquid cultures were serially diluted and spotted on solid selection media as outlined above.

\section{Monosaccharide analysis of the yeast cell wall}

Sample extraction and preparation procedures were undertaken according to previously described methods employing TFA hydrolysis [27]. Cultures $(50 \mathrm{~mL})$ were grown until $\mathrm{OD}=$ 1.0 to 1.2 and cells harvested by centrifugation at $2000 \mathrm{x}$ g for $5 \mathrm{~min}$. Cells were disrupted in 0.5 $\mathrm{mL}$ of $10 \mathrm{mM}$ Tris- $\mathrm{HCl}(\mathrm{pH} 8)$ using glass beads and a vortex. Cell walls were collected by centrifugation ( $3800 \mathrm{x}$ g for $5 \mathrm{~min}$ ) and washed in cold distilled water and dried in a vacuum concentrator. Cell wall pellets were hydrolyzed with $1 \mathrm{~mL}$ of $2 \mathrm{~N}$ trifluoroacetic acid (TFA) at $100{ }^{\circ} \mathrm{C}$ for 4 hours. Samples were lyophilized and re-suspended in $1 \mathrm{~mL}$ water prior to analysis. Monosaccharide composition was performed using High Performance Anion Exchange Chromatography on a Dionex ICS 3000 equipped with a pulse amperometric detector as previously described [28]. The monosaccharide composition of yeast samples was calculated by linear regression from a five point standard curve comprising glucose, mannose and glucosamine loaded before, during and after the sample set.

\section{RNA extraction and RT-PCR}

Total RNA was isolated from yeast strains using YeaStarTM RNA Kit (Zymo Research). Approximately $300 \mathrm{ng}$ of total RNA was treated with DNase (Invitrogen) and used as template for cDNA synthesis by SuperScript III Reverse Transcriptase (Invitrogen). PCR was undertaken using Taq 2X Master Mix (New England Biolabs Inc.) using conditions as provided by the manufacturer. The RT-PCR of apyrase transcripts was undertaken with attB1 and attB2 primers (Table S1). The yeast UBC6 gene (ubiquitin-conjugating enzyme) was used as a control.

\section{Immunoblotting}

Total protein was isolated from overnight yeast cultures as previously described [29]. The protein was quantified by Bradford (Thermo Scientific) [30]. A total of $5 \mu \mathrm{g}$ total protein was resuspended in $0.2 \mathrm{M}$ Tris-HCl, $\mathrm{pH} 6.5,8 \%$ (w/v) SDS, $8 \%$ (v/v) 2-mercaptoethanol, $40 \%$ (v/v) glycerol, and $0.04 \%(\mathrm{w} / \mathrm{v})$ bromophenol blue and boiled for $5 \mathrm{~min}$. Samples were subjected to $10 \%(\mathrm{w} / \mathrm{v})$ SDS-PAGE and blotted onto PVDF membrane. Heterologous expressed proteins were detected using the Universal antibody (UNI) against the Gateway ${ }^{\circledR}$ attB2 site [29], followed by incubation with a secondary antibody and detection by chemiluminescence using the Protein Detector ${ }^{\mathrm{TM}}$ LumiGLO ${ }^{\circledR}$ Western Blotting Kit (KPL Inc).

\section{Yeast microsomal preparations}

The yeast membranes were isolated from the complemented $\Delta g d a 1 \Delta y n d 1 \mathrm{dKO}$ yeast strains by initial disruption with glass beads in $400 \mu \mathrm{L}$ of chilled extraction buffer $(20 \mathrm{mM}$ Tris- $\mathrm{HCl}, 10$ $\mathrm{mM} \mathrm{MgCl} 2,1 \mathrm{mM}$ EDTA, $5 \%$ (v/v) Glycerol, $1 \mathrm{mM}$ DTT, $1 \mathrm{mM}$ PMSF and $1 \mathrm{x}$ Roche cOmplete Protease Inhibitor Cocktail). The cells were centrifuged at $5000 \mathrm{xg}$ for $10 \mathrm{~min}$ at $4{ }^{\circ} \mathrm{C}$ and supernatants collected. The supernatant was centrifuged at $50000 \mathrm{x} g$ for 1 hour at $4{ }^{\circ} \mathrm{C}$ and 
the resultant membrane pellet was re-suspended in $10 \mathrm{mM}$ Tris buffer ( $\mathrm{pH}$ 7.5) for the NTPDase assay.

\section{Measurement of NTPDase activity}

A total of $50 \mu \mathrm{g}$ microsomal protein was incubated in $500 \mu 1$ reaction buffer $(3 \mathrm{mM}$ NDP or NTP or NMP [Sigma-Aldrich], $3 \mathrm{mM} \mathrm{MnSO4,} 30 \mathrm{mM}$ Tris-MES, pH 6.5, and $0.03 \%$ (v/v) Triton X100 ) for $1 \mathrm{~h}$ at room temperature. The released phosphate was measured using the Malachite Green Phosphate Assay (ScienCell Research Laboratories) with slight modifications, namely that $100 \mu \mathrm{L}$ of reagent $\mathrm{A}$ and $100 \mu \mathrm{L}$ of reagent $\mathrm{B}$ were each added to the $50 \mu \mathrm{L}$ solution. The incubation times were undertaken according to the protocol.

\section{Particle Bombardment}

Plasmid DNA was isolated using QIAprep Spin Miniprep Kit (Qiagen). Particle bombardments for transient localizations were conducted according to previous methods [23]. Essentially, 0.6 $\mu \mathrm{g}$ plasmid DNA was added to a $25 \mu \mathrm{L}$ microcarrier/glycerol solution containing $400 \mu \mathrm{g}$ of microcarriers ( $1 \mu \mathrm{m}$ gold, Bio-Rad). Followed by $25 \mu \mathrm{L}$ of $2.5 \mathrm{M} \mathrm{CaCl}_{2}$ and $10 \mu \mathrm{L}$ of $0.1 \mathrm{M}$ spermidine. The solution was mixed for $10 \mathrm{~min}$ at $3000 \mathrm{rpm}$ and supernatant removed. The pellet was washed with $100 \%(\mathrm{v} / \mathrm{v})$ ethanol and re-suspended in $20 \mu \mathrm{L}$ of $100 \%(\mathrm{v} / \mathrm{v})$ ethanol, loaded onto a macrocarrier and air dried. The macrocarrier was placed onto the hepta adapter macrocarrier holder (leaving the other 6 empty). Fresh epidermal peels from yellow onions or whole Arabidopsis rosettes harvested from 6 to 8 -week old plants were bombarded under vacuum $(28 \mathrm{inHg})$ at a target distance of $6 \mathrm{~cm}$ and a helium pressure of $1100 \mathrm{psi}$. Arabidopsis rosettes were bombarded on $1 \%(\mathrm{w} / \mathrm{v})$ agar plates containing half strength Murashige and Skoog basal salt mixture. After bombardment, plant material were kept on plates overnight in the dark until imaging by confocal microscopy [23].

\section{Phylogenetic Analysis and Informatics}

The Arabidopsis apyrase protein sequences were obtained from The Arabidopsis Information Resource [22]. The human and plant apyrase protein sequences were obtained from GenBank [31] while yeast sequences were from the Saccharomyces Genome Database [32]. Phylogenetic trees were created using MEGA6 [33], with sequences aligned using MUSCLE (using UPGMB and default parameters), phylogenetic reconstruction was undertaken using Maximum Likelihood with 1000 Bootstrap Replications. Protein domains were obtained from InterProScan [34] and predicted transmembrane helices from TMHMM [35]. Protein features were visualized using DOG (Domain Graph, version 1.0) [36].

\section{GenBank Accessions}

The following sequences have been deposited at GenBank: AtAPY1/At3g04080 (JQ937231);

AtAPY2/At5g18280 (JQ937238); AtAPY3/At1g14240 (JF830008); AtAPY4/At1g14230

(JF830009); AtAPY5/At1g14250 (JF830010); AtAPY6/At2g02970 (JF830011); AtAPY7/At4g19180 (JQ965809). 


\section{Results}

\section{The Apyrase family of Arabidopsis thaliana}

A total of seven loci have been identified in the Arabidopsis genome that contain the apyrase domain, namely AtAPY1 (At3g04080), AtAPY2 (At5g18280), AtAPY3 (At1g14240), AtAPY4 (At1g14230), AtAPY5 (At1g14250), AtAPY6 (At2g02970) and AtAPY7 (At4g19180). A recent phylogenetic analysis of several hundred plant apyrases indicated that they fall into three major clades [14]. The seven member Arabidopsis apyrase family contain representatives in each clade and are clustered into the AtAPY1-2 clade I (GDA1-like), the AtAPY3 to 6 (clade II) and AtAPY7 in clade III (Figure 1A).

Since the eight Homo sapiens (human) apyrase genes (NTPDase1 to 8) have been characterized both biochemically and genetically [1] and the two apyrase enzymes from yeast, (GDA1 and YND1) have been extensively characterized [6], we undertook a phylogenetic analysis with several plant apyrases, the human apyrase family and the two yeast enzymes (Figure 1B). The clade I (GDA-like) Arabidopsis members (AtAPY1 and AtAPY2) form a distinct clade with the other characterized plant apyrases, human apyrases and the yeast GDA1 enzyme (Figure 1B). Although NTPDase6, GDA1, AtAPY1 and AtAPY2 appear to have a substrate preference for NDPs $[5,18,37]$, a number of the plant apyrases in this clade have been associated with exhibiting NTPase activity, namely StAPY3 [38] and PsAPY2 [39]. In humans, NTPase activity is associated with the secreted ecto-apyrase clade members (NTPDase1 to 3 and NTPDase8) and all display Type IV-A membrane protein topology (Figure S2). In contrast, plant members of the GDA-like clade are typical Type II membrane proteins (Figure 1A). The Arabidopsis AtAPY7 is only weakly associated with the human ecto-apyrase clade, it has a similar membrane topology and can also be classed as a Type IV-A membrane protein (Figure 1A).

The final group of Arabidopsis apyrase members forms an independent cluster (clade II) comprising AtAPY3 to 6. The apyrase members AtAPY3, AtAPY4 and AtAPY5 are recurrent tandem gene duplications on chromosome 1. All three contain a single putative $\mathrm{N}$-terminal transmembrane domain typical of Type II membrane proteins. In contrast, AtAPY6 (clade II) would appear to be Type IV-A membrane protein (Figure 1A).

\section{Disruption of the yeast apyrases GDA1 and YND1 affects the yeast cell wall composition}

The the $\triangle y n d 1 \Delta g d a 1$ strain was previously created by crossing the $\triangle y n d 1: \because U R A 3$ haploid (XGY4) with the $\triangle g d a 1: \angle E U 2$ haploid (G2-11). The $\triangle y$ nd1 $\triangle g d a 1$ dKO cells (KAI1) showed slow growth compared to the single mutants and the wild-type strain on YPAD plates at $30^{\circ} \mathrm{C}$ [6]. However, the laboratory that created this $\Delta y n d 1 \Delta g d a 1$ line has lost the original strain. We sought to recreate the $\triangle y$ nd $1 \Delta g d a 1$ strain by replacing the GDA1 open reading frame (ORF) with the URA ORF by a heterologous exchange in the $\Delta y n d 1$ single mutant background $[25,40]$. The $\Delta y$ d1 1 gda1 double knock-out ( $\mathrm{dKO})$ was verified using primer sets designed to assess the presence of the GDA ORF (Figure 2A). Only the URA ORF was detected by PCR in the $\Delta y n d 1 \triangle g d a 1 \mathrm{dKO}$ strain indicating that the URA ORF had successfully replaced the GDA1 ORF in the $\Delta y$ nd 1 single mutant background (Figure 2A).

The yeast cell wall contains $\beta(1 \rightarrow 3)$-D-glucan, $\beta(1 \rightarrow 6)$-D-glucan, chitin, and mannoproteins which are mostly synthesized at the plasma membrane [41]. Cell wall mannoproteins are 
synthesized in the ER/Golgi lumen and are dependent on the delivery of GDP-mannose from the cytosol, a process that is driven by the co-transport of GMP generated by apyrases (GND1/YND1) in the ER/Golgi lumen [42]. We analyzed the monosaccharide composition of a TFA hydrolyzed insoluble fraction extracted from the $\Delta y n d 1 \Delta g d a 1 \mathrm{dKO}$ strain after growth in modified YNB media after 2 days at $30^{\circ} \mathrm{C}$. The composition of mannose in this insoluble fraction was about $50 \%$ less than that found in wild-type (BY4741) cells (Figure 2B). The $\triangle y n d 1 \triangle$ gda1 $\mathrm{dKO}$ strain also contained less cell wall material including non-lumenal derived polymers, namely $\beta(1 \rightarrow 3)$-D-glucan, $\beta(1 \rightarrow 6)$-D-glucan, and $\mathrm{N}$-acetylglucosamine from chitin (Figure $2 \mathrm{C}$ ); highlighting the importance of the mannoprotein component in the construction of the yeast cell wall. Finally, similar to previously reported results [6], the newly generated $\triangle y n d 1 \Delta$ gda $1 \mathrm{dKO}$ also exhibits very slow growth on modified YNB media (data not shown).

\section{Yeasts lacking endogenous apyrase activity are complemented by human endo-apyrases} The human apyrases represent a biochemically well characterized family of enzymes with varying subcellular locations and activities. The members of the human apyrase family comprise the ER lumenal GDA-like NDPases (e.g. NTDPase6), an intracellular membrane associated clade with NDPase/NTPase activity (e.g. NTPDase7) and the ecto-apyrase group with NTPase activities (e.g. NTPDase1). We were interested in assessing the ability of these defined classes of apyrases to complement the $\Delta y n d 1 \Delta g d a 1 \mathrm{dKO}$ yeast strain generated above. The GDA-like human NTDPase6, with a substrate specificity for NDPs, was able to recover the growth phenotype observed in the $\Delta y n d 1 \Delta g d a 1 \mathrm{dKO}$ strain (Figure 3A). Neither the NTPDase1 (ectoapyrase) nor the NTPDase7 (intracellular with reported NDPase/NTPase activities) were able to complement the growth phenotype exhibited by the $\Delta y n d 1 \Delta g d a 1 \mathrm{dKO}$ strain (Figure $3 \mathrm{~A}$ ). NTPDase1 has a mixed NTP/NDP substrate specificity [43], while NTPDase7 has a preference for NTPs [44]. The presence of the yeast codon optimized human apyrase gene transcripts was verified by RT-PCR (Figure S3). Overall, these results indicate that subcellular context as well as substrate specificity are necessary for complementation of this yeast $\Delta y n d 1 \Delta g d a 1 \mathrm{dKO}$ strain.

\section{Yeast lacking endogenous apyrases can be complemented by Arabidopsis apyrases}

In order to assess the in vivo activities of the Arabidopsis apyrase family, we performed a complementation assay in the $\Delta y n d 1 \Delta g d a 1 \mathrm{dKO}$ as described above. Previously, the Arabidopsis clade I apyrase members AtAPY1 and AtAPY2 were shown to act as lumenal NDPases through the independent complementation of the glycosylation phenotype associated with the $\Delta g d a 1$ mutant background as well as the hygromycin sensitivities of the $\Delta y n d 1$ mutant related defects in the cell wall [18]. When these clade I Arabidopsis apyrases were expressed in the $\Delta y n d 1 \Delta g d a 1$ dKO background, both AtAPY1 and AtAPY2 were able to complement the growth phenotype when compared to yeast harboring the empty vector (pDR-Leu, Figure 3A). These results support our previous findings that both AtAPY 1 and AtAPY2 are able to function as internal Golgi lumenal NDPases. The heterologous expression of the clade II Arabidopsis apyrase members (AtAPY3 to 6) in the $\Delta y n d 1 \Delta g d a 1 \mathrm{dKO}$ background revealed that AtAPY4, AtAPY5 and AtAPY 6 were all able to complement the growth defect phenotype of the $\Delta y n d 1 \Delta g d a 1 \mathrm{dKO}$ yeast strain (Figure 3A), demonstrating these enzymes are also able to function as internal Golgi lumenal NDPases. In contrast, AtAPY3 exhibited relatively weak complementation compared to other members of this clade (Figure 3A). The clade III Arabidopsis apyrase AtAPY7 was unable to complement the growth phenotype of the $\Delta y n d 1 \Delta g d a 1 \mathrm{dKO}$ strain (Figure $3 \mathrm{~A}$ ). 
An analysis of the monosaccharide composition of the insoluble cell wall fractions from the complemented $\Delta y n d 1 \Delta g d a 1 \mathrm{dKO}$ strains further supported a role for the Arabidopsis apyrases as lumenal NDPases (Figure 3B). The proportion of mannose in cell wall extracts significantly increased in all the complemented strains with the AtAPY5 construct resulting in near wild-type levels (Figure 2B). The AtAPY3 construct was the least able to recover cell wall mannose, reflecting the reduced growth phenotype. The AtAPY4 construct resulted in only a marginal increase in cell wall mannose compared to AtAPY3, but was very capable of complementing the growth phenotype (Figure 3A). The ability to recover mannose in cell wall extracts of the $\Delta y n d 1 \Delta g d a 1 \mathrm{dKO}$ yeast likely reflects the activity of each apyrase with respect to the substrate GDP, (derived from lumenal GDP-mannose). Cell extracts were not analyzed from cells harboring the AtAPY7 construct as it exhibited no complementation of the growth phenotype.

To ensure the Arabidopsis apyrases were being adequately expressed in the $\Delta y n d 1 \Delta g d a 1 \mathrm{dKO}$ background, we analyzed protein extracts by immunoblotting. Evidence for the expression of constructs containing AtAPY1 to 5 in the $\Delta y n d 1 \Delta g d a 1 \mathrm{dKO}$ background was apparent (Figure S4). A faint band was detected for AtAPY 6 when $25 \mu \mathrm{g}$ microsomal protein was analyzed by immunoblotting and indicated some full-length product, however no evidence for the AtAPY 7 protein could be obtained. Previously we had observed processing of the AtAPY1 construct when expressed of in the $\Delta g d a 1$ background [18]. In this instance it is possible that the Cterminal is processed from AtAPY6 and AtAPY7. As a consequence we undertook RT-PCR analysis to verify the presence of all apyrase transcripts in the $\Delta y n d 1 \Delta g d a 1 \mathrm{dKO}$. Evidence for the presence of all transcripts was apparent for all constructs (Figure S3).

\section{The Arabidopsis AtAPY1 to 6 exhibit apyrase-like activities}

Microsomal preparations from the seven Arabidopsis apyrase members expressed in the $\Delta y n d 1 \Delta g d a 1 \mathrm{dKO}$ strain were used to measure latent NTPDase activity via inorganic phosphate release using malachite green [18]. The GDA-like clade I members AtAPY1 and AtAPY2 exhibited a clear preference towards the nucleotide substrates UDP $\left(0.7 \mu \mathrm{mol} \mathrm{Pi} \mathrm{h}{ }^{-1} \mu \mathrm{g}^{-1}\right)$ and $\mathrm{UDP} / \mathrm{GDP}$ ( 1 to $3 \mu \mathrm{mol} \mathrm{Pi} \mathrm{h}{ }^{-1} \mu \mathrm{g}^{-1}$ ) respectively (Figure 4), supporting previous reports indicating they both function as UDP/GDPases $[18,19]$. The clade II member AtAPY3 has a strong preference toward NTPs ( 8 to $12 \mu \mathrm{mol} \mathrm{Pi} \mathrm{h}^{-1} \mu \mathrm{g}^{-1}$ ) but also has significant activities toward ADP and GDP with 4 to $6 \mu \mathrm{mol} \mathrm{Pi} \mathrm{h}{ }^{-1} \mu \mathrm{g}^{-1}$ (Figure 4). In contrast, other members of the clade II apyrase family displayed an array of substrate preferences. No significant NTPase or NDPase activity could be detected for AtAPY4 except a slight affinity for CTP, while AtAPY5 demonstrated the highest level of NDP activity measured in our assay, ranging from 10 to 18 $\mu \mathrm{mol} \mathrm{Pi} \mathrm{h}{ }^{-1} \mu \mathrm{g}^{-1}$ (Figure 4). AtAPY6 appears to have a broad range of substrate activities toward all NTP and NDP substrates analyzed, with values from 0.5 to $2.5 \mu \mathrm{mol} \mathrm{Pi} \mathrm{h}^{-1} \mu \mathrm{g}^{-1}$ (Figure 4). Finally, the single clade III representative AtAPY7 displayed no detectable NTPase or NDPase activity under our experimental conditions (data not shown), although the presence of the protein could not be confirmed. In summary, the AtAPY1 to 6 Arabidopsis enzymes all exhibit classic apyrase-like NTPase and/or NDPases activities, with an absence of nucleotide monophosphate activity.

\section{Subcellular localization of the Arabidopsis apyrase family}

Previously, two members of the Arabidopsis apyrase family (AtAPY1 and AtAPY2) implicated as ecto-apyrases were shown to localize to Golgi membranes [17-19]. In an effort to resolve the 
401

subcellular distribution of Arabidopsis apyrases and subsequently their potential roles within the cell, we sought transiently co-localize all seven members using $\mathrm{N}$ - and C-terminal YFP fusions. As previously observed, both AtAPY1 and AtAPY2 localize to the cis-Golgi when either the Nor C-terminal YFP construct was used (Figure 5). Similarly cis-Golgi localization results were identified for AtAPY4, AtAPY5 and AtAPY7 using either the N- or C-terminal YFP constructs (Figure 5). These data indicate that AtAPY1, 2, 4, 5, 7 are likely cis-Golgi resident proteins. Neither the AtAPY3 nor the AtAPY6 constructs significantly overlapped with the cis-Golgi marker (Figure 5). The AtAPY3 C-terminal YFP construct resulted in an internal punctate signal with minimal cis-Golgi marker overlap. Further analysis with a trans-Golgi marker (CFP-VTI12) and an endosomal marker (CFP-RabF2a) indicted that AtAPY3 likely localizes to the endosome (Figure 6). Since the C-terminal AtAPY6 construct produced a diffuse web-like structure, we colocalized this construct using an ER marker (Figure 6). This resulted in a significant signal overlap indicating co-localization with the ER marker. Identical results were obtained by transient localizing in Arabidopsis rosette leaves by particle bombardments (Figure S5). The outcome of these localization experiments is summarized in Table 1. 


\section{Discussion}

The apyrase family of Arabidopsis would appear to be representative of plant species with members present in each phylogenetic clade [14]. Based on their subcellular distributions, the seven members of the Arabidopsis apyrase family are endo-apyrases. Their subcellular distributions are remarkably similar to the human endo-apyrase members with the majority localized to the Golgi apparatus, a single ER localized candidate and a single member localized to an intracellular vesicle (Figure 7). Our biochemical analysis indicated a wide range of substrate preference for most members of the family, providing evidence for functional diversity.

\section{The yeast and human apyrase families}

The recreation of the $\Delta y n d 1 \Delta g d a 1 \mathrm{dKO}$ strain enabled an investigation of the cell wall of yeast lacking any substantive lumenal apyrase activity. The results indicated that only the mannose content derived from mannoproteins was being substantially affected, with some compensation by D-glucan and chitin occurring. Similar observations have been made with gda1 single mutant in Candida albicans where chitin levels were found to increase in their cell walls [45]. The reduced mannose content of the wall also appeared to affect the total amount of cell wall material, supporting an integrated process for the biosynthesis and construction of the yeast cell wall [41]. The elimination of apyrase activity in yeast did not completely prevent the production of cell wall derived mannose, indicating that the transport of GDP-Man was still able to occur, likely at a reduced rate, without the counter substrate GMP. This is in contrast to deletion of VRG4, the Golgi resident GDP-Man transporter from yeast, which is lethal [46], as is the VIG9 null mutant, the GDP-mannose pyrophosphorylase essential for the biosynthesis of GDP-Man [47]. Thus, it is possible that lumenal GDP (or some other molecule) is able to be utilized as a counter substrate to enable the delivery of some GDP-Man into the Golgi lumen.

The regeneration of the $\Delta y n d 1 \Delta g d a 1 \mathrm{dKO}$ strain enabled us to examine the function of human apyrase family members. We selected the well-characterized ecto-apyrase NTPDase1 to assess the complementation of the mutant yeast strain with a secreted apyrase. Although complementation of the yeast $\mathrm{dKO}$ mutant strain with human apyrases was not as strong as our results with Arabidopsis apyrases, there was clear complementation by Golgi localized human apyrase NTPDase6. Minimal complementation was observed for the vesicle localized human apyrase NTPDase7 or the ecto-apyrase NTPDase1 (Figure 3). These results indicate that subcellular localization and biochemical function are important components of endo-apyrase yeast complementation assays.

\section{Clade I Arabidopsis apyrases: AtAPY1 and AtAPY2}

The Arabidopsis apyrases AtAPY1 and AtAPY2 are related to yeast GDA1-like (Clade I) and are the most extensively characterized plant apyrases. While they have been implicated to function at the plasma membrane as ATPases and ADPases regulating ecto-ATP/ADP concentrations [16,49] recent evidence provides a distinct functional role in Arabidopsis for both AtAPY1 and AtAPY2, namely as endo-apyrases residing in the Golgi lumen with UDPase and GDPase activities $[18,19]$. We have now demonstrated that both enzymes exhibit a clear substrate preference for UDP, as would be expected for apyrases responsible for the turnover of UDP after glycosylation reactions within the Golgi lumen. 
Several plant apyrases associated with the GDA1-like clade have been implicated as ectoapyrases through their association with NTPase activity and apoplastic localizations [38, 39]. However, since the human ecto-apyrase NTPDase 1 appears to require glycosylation for NTDPase activity [48] progression through the secretory pathway to provide glycan maturity and NTDPase function may be required. Whether this is also a feature of plant apyrases is currently unknown, but the fact that AtAPY1 and AtrAPY2 are both able to complement endo-apyrase activity in yeast, localize to the cis-Golgi and possess NDPase activities in vitro, it would indicate a central role for these enzymes as Arabidopsis endo-apyrases involved in the conversion of NDPs to NMPs as an important component of endomembrane glycosylation as previously discussed $[18,19]$.

\section{Clade II Arabidopsis apyrases: AtAPY3, AtAPY4 and AtAPY5}

The Arabidopsis clade II apyrase members have a diverse topology, with AtAPY3 to 5 exhibiting a single putative N-terminal transmembrane domain, while AtAPY6 appears to possess both an $\mathrm{N}$ - and $\mathrm{C}$ - terminal transmembrane domain. The mixed topology for this clade is not unique to Arabidopsis, with examples in both Glycine max and Vitis vinifera to name a few [14]. AtAPY3, AtAPY4 and AtAPY5 occur as recurrent tandem duplications and share 68\% identity, all three are expressed during Arabidopsis development with AtAPY3 predominately in the roots and both AtAPY4/AtAPY5 in the vegetative rosette [50]. Based on this information, it may be possible to speculate that these enzymes undertake similar functions at different developmental stages. However, the biochemical and localization analyses would support a more varied functional role in Arabidopsis.

The AtAPY3-YFP construct localized to small punctate structures with minimal overlap to the cis-Golgi or trans-Golgi markers, however there was considerable overlap with the late endosomal marker, RabF2a [51, 52]. This non-Golgi localization was recently confirmed in Nicotiana benthamiana [53]. The enzyme exhibited a clear substrate preference for NTPs and was unable to successfully complement the yeast $\Delta g d a 1 \Delta y n d 1 \mathrm{dKO}$ strain, which could have been due to poor expression or high protein turnover (Figure S4). Among the reported intracellular human NTPDases (NTPDase4, 5, 6 and 7), only NTPDase7 displays a strong preference for NTPs and is also reported to localize to internal vesicles [44]. However, no specific functional role for NTPDase 7 has been reported [1]. Yeast complementation involving NTPDase 7 also resulted in poor growth of the $\Delta g d a 1 \Delta y n d 1 \mathrm{dKO}$ yeast strain. While similar results were observed for AtAPY3, it is possible that subcellular localization played an important role in these experiments. Given its NTP preference and subcellular localization, a possible role in intra-cellular signaling through an involvement with the GTP-binding/GTPase regulatory networks [54] or NTP secretion [55] would be conceivable.

In contrast, both AtAPY 4-cYFP and nYFP-AtAPY5 localized to the cis-Golgi and their coding regions were able to complement the yeast $\Delta g d a 1 \Delta y n d 1$ strain. AtAPY5 exhibited the highest specific activities for NDPs of all the Arabidopsis apyrases, which resulted in the high mannose yield from cell wall extracts of complemented yeast strain. The biochemical analysis of AtAPY4 resulted in the lowest NDPase activates measured, exhibiting a substrate preference for CTP. However, even with this reduced NDPase activity, its localization to the Golgi lumen likely assisted in the positive complementation phenotype in yeast $\Delta g d a 1 \Delta y n d 1$ cells. Overall, these results suggest in planta endo-apyrase roles for these enzymes with functional roles related to 
their NTPDase activities which could constitute lumenal NDPase activity during specific aspects of vegetative growth. Whether AtAPY4 also functions as a lumenal NTPase requires further investigations.

\section{The clade II Arabidopsis apyrase: AtAPY6}

Similar to AtAPY6, all four human ecto-apyrases are reported to contain both N- and C-terminal transmembrane domains. Based on these structural characteristics, we initially considered AtAPY6 a potential ecto-apyrase. However, AtAPY6-YFP constructs were localized to the ER, biochemical assays indicate broad NDP/NTP substrate preferences and its heterologous expression in yeast complemented the $\Delta g d a 1 \Delta y n d 1$ strain, resulting in a high amount of mannose recovered from cell wall extracts. In contrast, the human ecto-apyrase NTPDase1 was unable to successfully complement the yeast mutant strain when compared to results from the human GDA1-like apyrase, NTPDase6.

\section{Similar to other members of clade II, AtAPY6 exhibits a defined expression pattern during} Arabidopsis development, namely mature pollen [50]. A recent analysis of AtAPY6 confirmed its high expression in mature pollen and an analysis of atapy 6 mutants indicated a minor role in pollen development associated with abnormal exine patterning [21]. The localization of AtAPY6 to the ER and its broad substrate specify is unique amongst the Arabidopsis apyrase family. The only other ER localized apyrase is the human NTPDase5, which is thought to remove the inhibiting effects of UDP and support the efficient re-glucosylation of proteins enabling correct folding of glycoproteins [56]. These observations in combination with the biochemical and molecular data would support an endo-apyrase role for AtAPY6. Finally with its ER localization, it may have a role in supporting glucosylation of nascent $N$-glycans [57] through the turnover of UDP; inhibition of which is most evident in maturing pollen [21].

\section{Clade III Arabidopsis apyrase: AtAPY7}

The final member of the seven apyrase-like proteins encoded by Arabidopsis and the only member of clade III is AtAPY7. While localization of the AtAPY7-YFP construct seems to indicate cis-Golgi localization, the construct was unable to complement the $\Delta g d a 1 \Delta y n d 1$ yeast strain. Furthermore, biochemical analysis of microsomal fractions showed no NTPDase activity. Although it is possible that the heterologous expression in yeast was unsuccessful, we were able to detect AtAPY7 transcripts in the transformed yeast cells. The AtAPY7 protein sequence contains the well characterized five apyrase conserve regions (ACRs) indicating it is a member of the apyrase family [21].

A recent molecular analysis of AtAPY7 determined that it was ubiquitously expressed in a range of Arabidopsis tissues and developmental stages. An analysis of atapy7 mutants also indicated minor aberrations to the pollen exine as observed in atapy 6 mutants. Interestingly, dKO mutants lacking both AtAPY6 and AtAPY7 produced relatively normal plants but with low male fertility from collapsed pollen which further resulted in reduced seed set [21]. Given the likelihood that AtAPY7 does not appear to function as a typical apyrase, it is difficult to explain the synergistic effects observed in atapy6atapy7 lines. The expression pattern for AtAPY7 would not indicate a specific role in pollen development, indicating its function could be associated with an important lumenal process which is in demand during pollen maturation. A more detailed analysis of AtAPY7 function needs to be undertaken to determine its role in pollen development. 
554

555

556

557

558

559

560

561

562

563

564

565

566

567

568

569

Overall our results would indicate that at least for the reference plant Arabidopsis, all members of the apyrase family are localized internally within the endomembrane system. Although there is evidence that individual apyrase members from other plant species can be secreted, we saw no evidence for this with Arabidopsis apyrase family members. Indeed, biochemical evidence and yeast complementation experiments would suggest an overall preference for NDPs over NTPs. These findings along with a number of recent studies $[18,19,58]$ do not necessarily preclude the existence of an ecto-apyrase in Arabidopsis, however they do indicate that further investigations are required. Interestingly, many of the characterized plant ecto-apyrases are encoded by legume species and appear to have roles in host-pathogen interactions such as nodulation [59]. Since the extracellular space is where symbiotic interactions initially occur, it is possible that apyrases from legume species have evolved to undertake ecto-apyrase functions associated with these interactions. Given Arabidopsis is incapable of forming such nitrogen fixing associations, it is conceivable that this function never evolved in certain plant lineages. However, further is necessary to determine whether this is the case. 


\section{Author Contribution}

571 Tsan-Yu Chiu, Dominique Loqué and Joshua L. Heazlewood designed the experiment. Tsan-Yu 572 Chiu, Jeemeng Lao and Bianca Manalansan performed the experiments. Tsan-Yu Chiu, Joshua 573 L. Heazlewood, Dominique Loqué and Stanley J. Roux analyzed the data. Tsan-Yu Chiu, and 574 Joshua L. Heazlewood wrote the manuscript.

\section{Acknowledgments} The vector pRS416-GPD was kindly provided by Dr. Arlen Johnson (University of Texas, Austin). We would also like to thank Huu Tran and Dr. Suzan Yilmaz (Joint BioEnergy Institute) for advice and assistance with assays.

\section{Funding} Research, of the U.S. Department of Energy [DE-AC02-05CH11231] and an Australian Research Council Future Fellowship [JLH FT130101165]. 
587

588

589

590

591

592

593

594

595

596

597

598

599

600

601

602

603

604

605

606

607

608

609

610

611

612

613

614

615

616

617

618

619

620

621

622

623

624

625

626

627

628

629

630

631

\section{References}

1 Knowles, A. F. (2011) The GDA1_CD39 superfamily: NTPDases with diverse functions. Purinergic signalling. 7, 21-45

2 Leal, D. B., Streher, C. A., Neu, T. N., Bittencourt, F. P., Leal, C. A., da Silva, J. E., Morsch, V. M. and Schetinger, M. R. (2005) Characterization of NTPDase (NTPDase1; ectoapyrase; ecto-diphosphohydrolase; CD39; EC 3.6.1.5) activity in human lymphocytes. Biochim. Biophys. Acta. 1721, 9-15

3 Enjyoji, K., Sevigny, J., Lin, Y., Frenette, P. S., Christie, P. D., Esch, J. S. A., Imai, M., Edelberg, J. M., Rayburn, H., Lech, M., Beeler, D. L., Csizmadia, E., Wagner, D. D., Robson, S. C. and Rosenberg, R. D. (1999) Targeted disruption of cd39/ATP diphosphohydrolase results in disordered hemostasis and thromboregulation. Nat. Med. 5, 1010-1017

4 Wang, T. F. and Guidotti, G. (1998) Golgi localization and functional expression of human uridine diphosphatase. J. Biol. Chem. 273, 11392-11399

5 Abeijon, C., Yanagisawa, K., Mandon, E. C., Hausler, A., Moremen, K., Hirschberg, C. B. and Robbins, P. W. (1993) Guanosine diphosphatase is required for protein and sphingolipid glycosylation in the Golgi lumen of Saccharomyces cerevisiae. J. Cell Biol. 122, 307-323

6 Gao, X. D., Kaigorodov, V. and Jigami, Y. (1999) YND1, a homologue of GDA1, encodes membrane-bound apyrase required for Golgi $N$ - and $\mathrm{O}$-glycosylation in Saccharomyces cerevisiae. J. Biol. Chem. 274, 21450-21456

7 Sánchez, R., Franco, A., Gacto, M., Notario, V. and Cansado, J. (2003) Characterization of $g d p 1^{+}$as encoding a GDPase in the fission yeast Schizosaccharomyces pombe. FEMS Microbiol. Lett. 228, 33-38

8 Clark, G. and Roux, S. J. (2011) Apyrases, extracellular ATP and the regulation of growth. Curr. Opin. Plant Biol. 14, 700-706

9 Jeter, C. R., Tang, W. Q., Henaff, E., Butterfield, T. and Roux, S. J. (2004) Evidence of a novel cell signaling role for extracellular adenosine triphosphates and diphosphates in Arabidopsis. Plant Cell. 16, 2652-2664

10 Song, C. J., Steinebrunner, I., Wang, X. Z., Stout, S. C. and Roux, S. J. (2006)

Extracellular ATP induces the accumulation of superoxide via NADPH oxidases in Arabidopsis. Plant Physiol. 140, 1222-1232

11 Kim, S. Y., Sivaguru, M. and Stacey, G. (2006) Extracellular ATP in plants.

Visualization, localization, and analysis of physiological significance in growth and signaling.

Plant Physiol. 142, 984-992

12 Clark, G., Fraley, D., Steinebrunner, I., Cervantes, A., Onyirimba, J., Liu, A., Torres, J., Tang, W., Kim, J. and Roux, S. J. (2011) Extracellular nucleotides and apyrases regulate stomatal aperture in Arabidopsis. Plant Physiol. 156, 1740-1753

13 Choi, J., Tanaka, K., Cao, Y., Qi, Y., Qiu, J., Liang, Y., Lee, S. Y. and Stacey, G. (2014) Identification of a plant receptor for extracellular ATP. Science. 343, 290-294

14 Clark, G. B., Morgan, R. O., Fernandez, M. P., Salmi, M. L. and Roux, S. J. (2014)

Breakthroughs spotlighting roles for extracellular nucleotides and apyrases in stress responses and growth and development. Plant Sci. 225, 107-116

15 Steinebrunner, I., Wu, J., Sun, Y., Corbett, A. and Roux, S. J. (2003) Disruption of apyrases inhibits pollen germination in Arabidopsis. Plant Physiol. 131, 1638-1647

16 Wu, J., Steinebrunner, I., Sun, Y., Butterfield, T., Torres, J., Arnold, D., Gonzalez, A., Jacob, F., Reichler, S. and Roux, S. J. (2007) Apyrases (nucleoside triphosphate- 
632

633

634

635

636

637

638

639

640

641

642

643

644

645

646

647

648

649

650

651

652

653

654

655

656

657

658

659

660

661

662

663

664

665

666

667

668

669

670

671

672

673

674

675

676

677

diphosphohydrolases) play a key role in growth control in Arabidopsis. Plant Physiol. 144, 961975

17 Parsons, H. T., Christiansen, K., Knierim, B., Carroll, A., Ito, J., Batth, T. S., SmithMoritz, A. M., Morrison, S., McInerney, P., Hadi, M. Z., Auer, M., Mukhopadhyay, A., Petzold, C. J., Scheller, H. V., Loqué, D. and Heazlewood, J. L. (2012) Isolation and proteomic characterization of the Arabidopsis Golgi defines functional and novel components involved in plant cell wall biosynthesis. Plant Physiol. 159, 12-26

18 Chiu, T. Y., Christiansen, K., Moreno, I., Lao, J., Loqué, D., Orellana, A., Heazlewood, J. L., Clark, G. and Roux, S. J. (2012) AtAPY1 and AtAPY2 function as Golgi-localized nucleoside diphosphatases in Arabidopsis thaliana. Plant Cell Physiol. 53, 1913-1925

19 Schiller, M., Massalski, C., Kurth, T. and Steinebrunner, I. (2012) The Arabidopsis apyrase AtAPY1 is localized in the Golgi instead of the extracellular space. BMC Plant Biol. 12, 123

20 Lim, M. H., Wu, J., Yao, J., Gallardo, I. F., Dugger, J. W., Webb, L. J., Huang, J., Salmi, M. L., Song, J., Clark, G. and Roux, S. J. (2014) Apyrase suppression raises extracellular ATP levels and induces gene expression and cell wall changes characteristic of stress responses. Plant Physiol. 164, 2054-2067

21 Yang, J., Wu, J., Romanovicz, D., Clark, G. and Roux, S. J. (2013) Co-regulation of exine wall patterning, pollen fertility and anther dehiscence by Arabidopsis apyrases 6 and 7 . Plant Physiol. Bioch. 69, 62-73

22 Lamesch, P., Berardini, T. Z., Li, D., Swarbreck, D., Wilks, C., Sasidharan, R., Muller, R., Dreher, K., Alexander, D. L., Garcia-Hernandez, M., Karthikeyan, A. S., Lee, C. H., Nelson, W. D., Ploetz, L., Singh, S., Wensel, A. and Huala, E. (2012) The Arabidopsis Information Resource (TAIR): improved gene annotation and new tools. Nucleic Acids Res. 40, D1202-1210 23 Lao, J., Oikawa, A., Bromley, J. R., McInerney, P., Suttangkakul, A., Smith-Moritz, A. M., Plahar, H., Chiu, T.-Y., González Fernández-Niño, S. M., Ebert, B., Yang, F., Christiansen, K. M., Hansen, S. F., Stonebloom, S., Adams, P. D., Ronald, P. C., Hillson, N. J., Hadi, M. Z., Vega-Sanchez, M. E., Loqué, D., Scheller, H.V. and Heazlewood, J. L. (2014) The plant glycosyltransferase clone collection for functional genomics. Plant J. 79, 517-529

24 MGC Project Team. (2009) The completion of the Mammalian Gene Collection (MGC). Genome Res. 19, 2324-2333

25 Baudin, A., Ozier-Kalogeropoulos, O., Denouel, A., Lacroute, F. and Cullin, C. (1993) A simple and efficient method for direct gene deletion in Saccharomyces cerevisiae. Nucleic Acids Res. 21, 3329-3330

26 Mumberg, D., Muller, R. and Funk, M. (1995) Yeast vectors for the controlled expression of heterologous proteins in different genetic backgrounds. Gene. 156, 119-122

27 Dallies, N., François, J. and Paque, V. (1998) A new method for quantitative determination of polysaccharides in the yeast cell wall. Application to the cell wall defective mutants of Saccharomyces cerevisiae. Yeast. 14, 1297-1306

28 Ebert, B., Rautengarten, C., Guo, X., Xiong, G., Stonebloom, S., Smith-Moritz, A. M., Herter, T., Chan, L. J., Adams, P. D., Petzold, C. J., Pauly, M., Willats, W. G., Heazlewood, J. L. and Scheller, H. V. (2015) Identification and Characterization of a Golgi-Localized UDP-Xylose Transporter Family from Arabidopsis. Plant Cell. 27, 1218-1227

29 Eudes, A., Baidoo, E. E., Yang, F., Burd, H., Hadi, M. Z., Collins, F. W., Keasling, J. D. and Loqué, D. (2011) Production of tranilast [N-(3',4'-dimethoxycinnamoyl)-anthranilic acid] and its analogs in yeast Saccharomyces cerevisiae. Appl. Microbiol. Biotechnol. 89, 989-1000 
30 Bradford, M. M. (1976) A rapid and sensitive method for the quantitation of microgram quantities of protein utilizing the principle of protein-dye binding. Anal. Biochem. 72, 248-254 31 Benson, D. A., Cavanaugh, M., Clark, K., Karsch-Mizrachi, I., Lipman, D. J., Ostell, J. and Sayers, E. W. (2013) GenBank. Nucleic Acids Res. 41, D36-D42 32 Cherry, J. M., Hong, E. L., Amundsen, C., Balakrishnan, R., Binkley, G., Chan, E. T., Christie, K. R., Costanzo, M. C., Dwight, S. S., Engel, S. R., Fisk, D. G., Hirschman, J. E., Hitz, B. C., Karra, K., Krieger, C. J., Miyasato, S. R., Nash, R. S., Park, J., Skrzypek, M. S., Simison, M., Weng, S. and Wong, E. D. (2012) Saccharomyces Genome Database: the genomics resource of budding yeast. Nucleic Acids Res. 40, D700-705

33 Tamura, K., Stecher, G., Peterson, D., Filipski, A. and Kumar, S. (2013) MEGA6: Molecular Evolutionary Genetics Analysis Version 6.0. Mol. Biol. Evol. 30, 2725-2729 34 Jones, P., Binns, D., Chang, H. Y., Fraser, M., Li, W. Z., McAnulla, C., McWilliam, H., Maslen, J., Mitchell, A., Nuka, G., Pesseat, S., Quinn, A. F., Sangrador-Vegas, A., Scheremetjew, M., Yong, S. Y., Lopez, R. and Hunter, S. (2014) InterProScan 5: genome-scale protein function classification. Bioinformatics. 30, 1236-1240

35 Krogh, A., Larsson, B., von Heijne, G. and Sonnhammer, E. L. (2001) Predicting transmembrane protein topology with a hidden Markov model: application to complete genomes. J. Mol. Biol. 305, 567-580

36 Ren, J., Wen, L., Gao, X., Jin, C., Xue, Y. and Yao, X. (2009) DOG 1.0: illustrator of protein domain structures. Cell Res. 19, 271-273

37 Hicks-Berger, C. A., Chadwick, B. P., Frischauf, A. M. and Kirley, T. L. (2000) Expression and characterization of soluble and membrane-bound human nucleoside triphosphate diphosphohydrolase 6 (CH39L2). J. Biol. Chem. 275, 34041-34045

38 Riewe, D., Grosman, L., Fernie, A. R., Wucke, C. and Geigenberger, P. (2008) The potato-specific apyrase is apoplastically localized and has influence on gene expression, growth, and development. Plant Physiol. 147, 1092-1109

39 Shibata, K., Morita, Y., Abe, S., Stankovic, B. and Davies, E. (1999) Apyrase from pea stems: Isolation, purification, characterization and identification of a NTPase from the cytoskeleton fraction of pea stem tissue. Plant Physiol. Bioch. 37, 881-888

40 Wach, A., Brachat, A., Pohlmann, R. and Philippsen, P. (1994) New heterologous modules for classical or PCR-based gene disruptions in Saccharomyces cerevisiae. Yeast. 10, 1793-1808

41 Orlean, P. (2012) Architecture and biosynthesis of the Saccharomyces cerevisiae cell wall. Genetics. 192, 775-818

42 Engel, J., Schmalhorst, P. S. and Routier, F. H. (2012) Biosynthesis of the fungal cell wall polysaccharide galactomannan requires intraluminal GDP-mannose. J. Biol. Chem. 287, 44418-44424

43 Christoforidis, S., Papamarcaki, T., Galaris, D., Kellner, R. and Tsolas, O. (1995) Purification and properties of human placental ATP-diphosphohydrolase. Eur. J. Biochem. 234, $66-74$

44 Shi, J. D., Kukar, T., Wang, C. Y., Li, Q. Z., Cruz, P. E., Davoodi-Semiromi, A., Yang, P., Gu, Y. R., Lian, W., Wu, D. H. and She, J. X. (2001) Molecular cloning and characterization of a novel mammalian endo-apyrase (LALP1). J. Biol. Chem. 276, 17474-17478

45 Herrero, A. B., Uccelletti, D., Hirschberg, C. B., Dominguez, A. and Abeijon, C. (2002) The Golgi GDPase of the fungal pathogen Candida albicans affects morphogenesis, glycosylation, and cell wall properties. Eukaryot. Cell. 1, 420-431 
46 Dean, N., Zhang, Y. B. and Poster, J. B. (1997) The VRG4 gene is required for GDPmannose transport into the lumen of the Golgi in the yeast, Saccharomyces cerevisiae. J. Biol. Chem. 272, 31908-31914

47 Yoda, K., Kawada, T., Kaibara, C., Fujie, A., Abe, M., Hitoshi, Hashimoto, Shimizu, J., Tomishige, N., Noda, Y. and Yamasaki, M. (2000) Defect in cell wall integrity of the yeast Saccharomyces cerevisiae caused by a mutation of the GDP-mannose pyrophosphorylase gene VIG9. Biosci. Biotechnol. Biochem. 64, 1937-1941

48 Wu, J. J., Choi, L. E. and Guidotti, G. (2005) $N$-linked oligosaccharides affect the enzymatic activity of CD39: Diverse interactions between seven $N$-linked glycosylation sites. Mol. Biol. Cell. 16, 1661-1672

49 Steinebrunner, I., Jeter, C., Song, C. and Roux, S. J. (2000) Molecular and biochemical comparison of two different apyrases from Arabidopsis thaliana. Plant Physiol. Bioch. 38, 913922

50 Winter, D., Vinegar, B., Nahal, H., Ammar, R., Wilson, G. V. and Provart, N. J. (2007) An "Electronic Fluorescent Pictograph" browser for exploring and analyzing large-scale biological data sets. PLOS ONE. 2, e718

51 Rutherford, S. and Moore, I. (2002) The Arabidopsis Rab GTPase family: another enigma variation. Curr. Opin. Plant Biol. 5, 518-528

52 Geldner, N., Denervaud-Tendon, V., Hyman, D. L., Mayer, U., Stierhof, Y. D. and Chory, J. (2009) Rapid, combinatorial analysis of membrane compartments in intact plants with a multicolor marker set. Plant J. 59, 169-178

53 Poulsen, C. P., Dilokpimol, A. and Geshi, N. (2015) Arabinogalactan biosynthesis: Implication of AtGALT29A enzyme activity regulated by phosphorylation and co-localized enzymes for nucleotide sugar metabolism in the compartments outside of the Golgi apparatus. Plant Signal. Behav. 10, e984524

54 Vernoud, V., Horton, A. C., Yang, Z. B. and Nielsen, E. (2003) Analysis of the small GTPase gene superfamily of Arabidopsis. Plant Physiol. 131, 1191-1208

55 Geisler, J. C., Corbin, K. L., Li, Q., Feranchak, A. P., Nunemaker, C. S. and Li, C. (2013) Vesicular nucleotide transporter-mediated ATP release regulates insulin secretion.

Endocrinology. 154, 675-684

56 Trombetta, E. S. and Helenius, A. (1999) Glycoprotein reglucosylation and nucleotide sugar utilization in the secretory pathway: identification of a nucleoside diphosphatase in the endoplasmic reticulum. EMBO J. 18, 3282-3292

57 Song, W., Henquet, M. G. L., Mentink, R. A., van Dijk, A. J., Cordewener, J. H. G., Bosch, D., America, A. H. P. and van der Krol, A. R. (2011) N-glycoproteomics in plants: Perspectives and challenges. J. Proteomics. 74, 1463-1474

58 Massalski, C., Bloch, J., Zebisch, M. and Steinebrunner, I. (2015) The biochemical properties of the Arabidopsis ecto-nucleoside triphosphate diphosphohydrolase AtAPY1 contradict a direct role in purinergic signaling. PLOS ONE. 10

59 Tanaka, K., Nguyen, C. T., Libault, M., Cheng, J. L. and Stacey, G. (2011) Enzymatic activity of the soybean ecto-apyrase GS52 is essential for stimulation of nodulation. Plant Physiol. 155, 1988-1998

60 Tanz, S. K., Castleden, I., Hooper, C. M., Vacher, M., Small, I. and Millar, A. H. (2013) SUBA3: a database for integrating experimentation and prediction to define the SUBcellular location of proteins in Arabidopsis. Nucleic Acids Res. 41, 1185-1191 
771

Figure 1. The apyrase family of Arabidopsis thaliana.

(A) Schematic protein structure of the seven Arabidopsis apyrase proteins outlining the apyrase conserved domain GDA1_CD39 and predicted transmembrane helices (TMD). The clade designations are based on plant sequences.

(B) Phylogenetic tree of Arabidopsis apyrase family with yeast, human and previously reported plant apyrases, including pea (PsAPY1 and PsAPY2), potato (StAPY3), soybean (GS52 and GS50) and Dolichos biflorus (DbLNP). The phylogenetic tree was created using MEGA6 using MUSCLE (1000 replicas). The percentage of replicate trees is shown on the branches. The bar indicates branch length.

Figure 2. Generation and cell wall analysis of $\Delta g d a 1 \Delta y n d 1$ yeast double knockout strain. (A) RT-PCR analysis of wild-type (BY4741), $\Delta y n d 1$ and $\Delta g d a 1 \Delta y n d 1$ yeast strains outlining the creation of the double knockout strain by replacing the GDA1 locus with URA3 by homologous recombination. Characterization of cell wall material (TFA hydrolyzed) by anion exchange chromatography from overnight cultures of wild-type (BY4741) and $\Delta$ gda1 $\Delta y n d 1$ yeast strains (B) expressed and mole (\%) (C) expressed as g/g fresh weight (FW) $(n=3 \pm$ SE).

Figure 3. Complementation of the $\Delta g d a 1 \Delta y n d 1$ yeast double knockout strain.

(A) Assessment of functional complementation of $\Delta g d a 1 \Delta y n d 1$ by growth complementation on YNB-Leu-Ura media using a serial dilution. The pDR-Leu is an empty vector control.

(B) Characterization of cell wall material in the $\Delta$ gda1 $\Delta y n d 1$ double knockout (dKO) line complemented by expressing Arabidopsis apyrases AtAPY1 to 6. Cell wall material from overnight cultures was hydrolyzed by TFA and analyzed by anion exchange chromatography. The $(*)$ indicates a significant difference in the mannose content $(p<0.01)$ between the complemented lines and the $\Delta g d a 1 \Delta y n d 1$ double knockout line $(n=3 \pm \mathrm{SE})$.

\section{Figure 4. Specific activity of the Arabidopsis apyrase enzymes AtAPY1 to 6.}

Latent NTPDase activity was assessed using isolated microsomes from overnight cultures of $\Delta g d a 1 \Delta y n d 1$ double knockout strain expressing the Arabidopsis apyrase genes. The NTP substrates are displayed as filled bars, while the NDP substrates are shown as empty bars. Activity is expressed in $\mu$ mole $\mathrm{Pi} /$ hour $/ \mu \mathrm{g}$ total protein $(n=3 \pm \mathrm{SE})$.

Figure 5. Subcellular localization of the Arabidopsis apyrase family.

Localization of transiently expressed Arabidopsis apyrase proteins using an N- or C-terminal Yellow Fluorescent Protein (YFP) using particle bombardment in onion epidermal cells. For each construct (three panels), the first contains the protein of interest (apyrase), the second contains the organelle marker and the third shows the overlay image. Subcellular localization of apyrase proteins was undertaken using either an N-terminal YFP (nYFP) or a C-terminal YFP (cYFP). The cis-Golgi marker was $\alpha$-mannosidase I fused to Cyan Fluorescent Protein (CFP). Scale $=10 \mu \mathrm{m}$.

\section{Figure 6. Subcellular localization of AtAPY3 and AtAPY6.}

The punctate structures identified using the AtAPY3-CFP construct was assessed using a transGolgi marker (CFP-VTI12) and an endosomal marker (CFP-RabF2a). Overlap in signal was 
816 observed with the endosomal marker (arrows). The ER localization of AtAPY6 was confirmed using the ER localization marker, WAK2-CFP-HDEL in combination with the AtAPY6-cYFP construct. Scale $=10 \mu \mathrm{m}$.

Figure 7. Schematic diagram summarizing the subcellular localization, putative topology and major specific activity of the Arabidopsis apyrase family. 
Table 1. Summary of subcellular localizations for the Arabidopsis apyrase family.

\begin{tabular}{|c|c|c|c|c|c|c|c|}
\hline AGI & Name & $\begin{array}{l}\text { Onion } \\
\text { (C-YFP) }\end{array}$ & $\begin{array}{l}\text { Onion } \\
\text { (N-YFP) }\end{array}$ & $\begin{array}{l}\text { Arabidopsis } \\
\text { (C-YFP) }\end{array}$ & $\begin{array}{l}\text { SUBA }^{1} \\
\text { (MS) }\end{array}$ & $\begin{array}{l}\text { SUBA }^{2} \\
\text { (FP) }\end{array}$ & $\begin{array}{l}\text { Inferred } \\
\text { Location }\end{array}$ \\
\hline AT3G04080.1 & AtAPY1 & cis-Golgi & cis-Golgi & cis-Golgi & Golgi & Golgi & cis-Golgi \\
\hline AT5G18280.1 & AtAPY2 & cis-Golgi & cis-Golgi & cis-Golgi & Golgi & Golgi & cis-Golgi \\
\hline AT1G14240.1 & AtAPY3 & endosome & - & endosome & 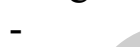 & & endosome \\
\hline AT1G14230.1 & AtAPY4 & cis-Golgi & - & cis-Golgi & & & cis-Golgi \\
\hline AT1G14250.1 & AtAPY5 & - & cis-Golgi & cis-Golgi & & - & cis-Golgi \\
\hline AT2G02970.1 & AtAPY6 & ER & - & ER & & - & ER \\
\hline AT4G19180.1 & AtAPY7 & cis-Golgi & cis-Golgi & cis-Golgi & & - & cis-Golgi \\
\hline
\end{tabular}

${ }^{1}$ Subcellular location by proteomic analyses as outlined in the SUBcellular Arabidopsis database [60].

${ }^{2}$ Subcellular location by fluorescently tagged protein as outlined in the SUBcellular Arabidopsis database [60] 
A

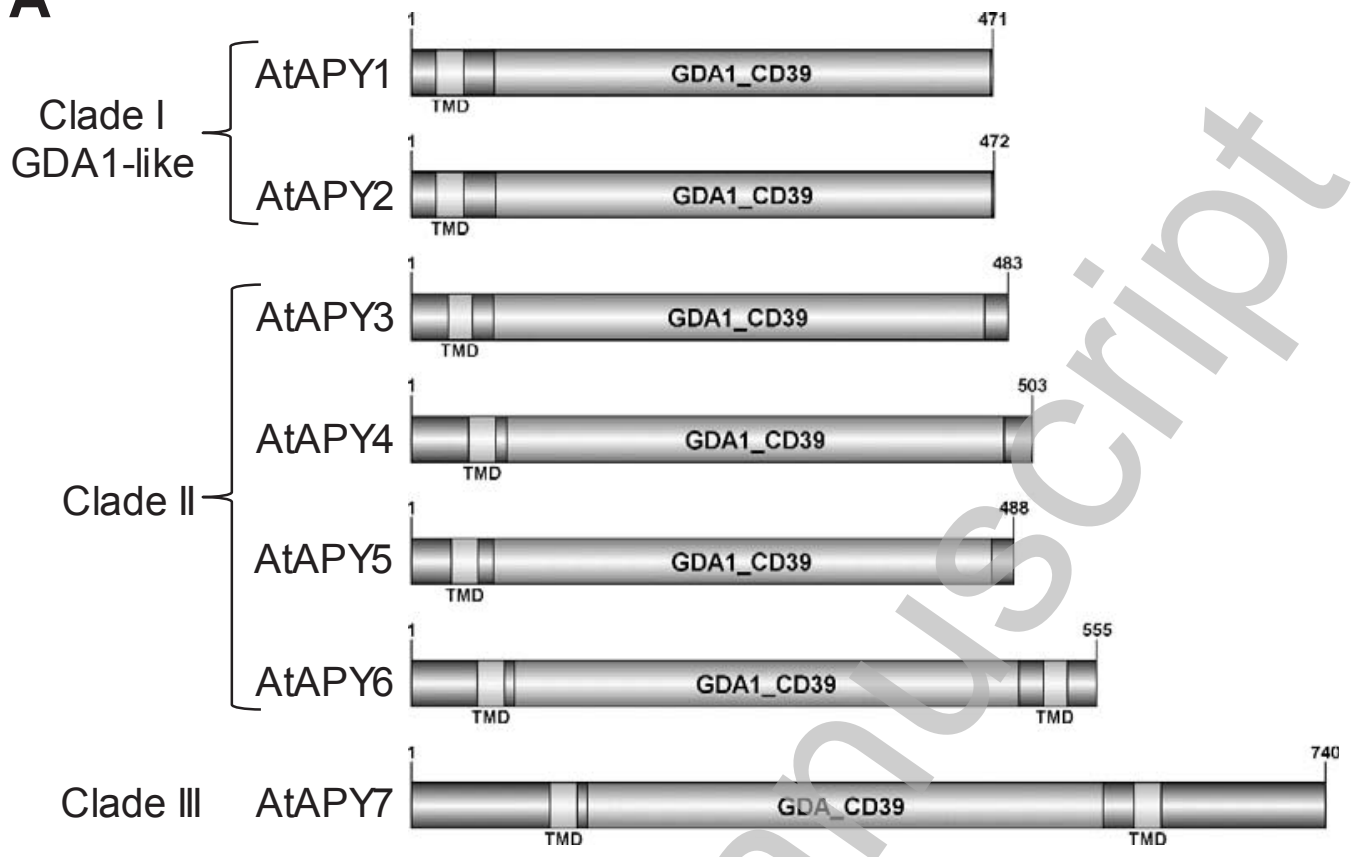

B

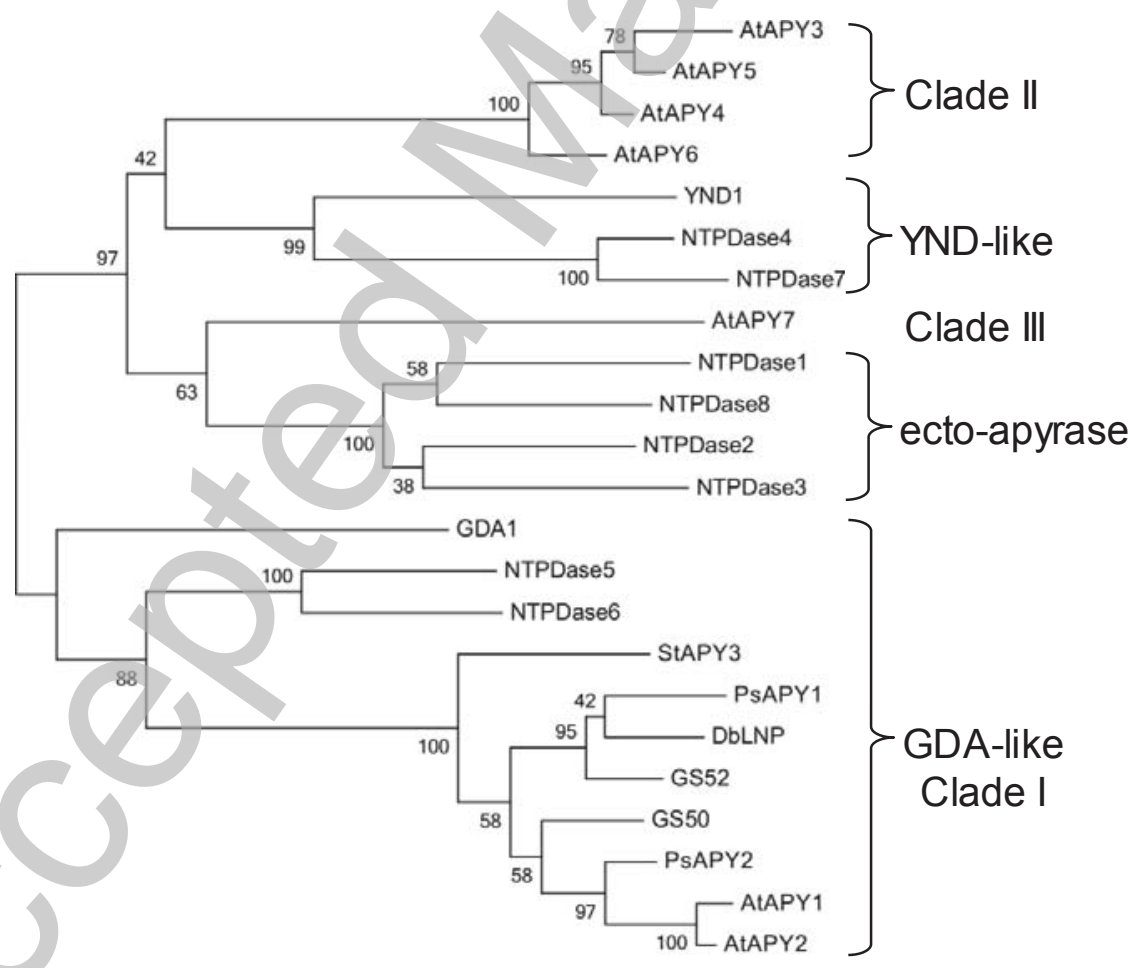

831 Figure 1.

832 


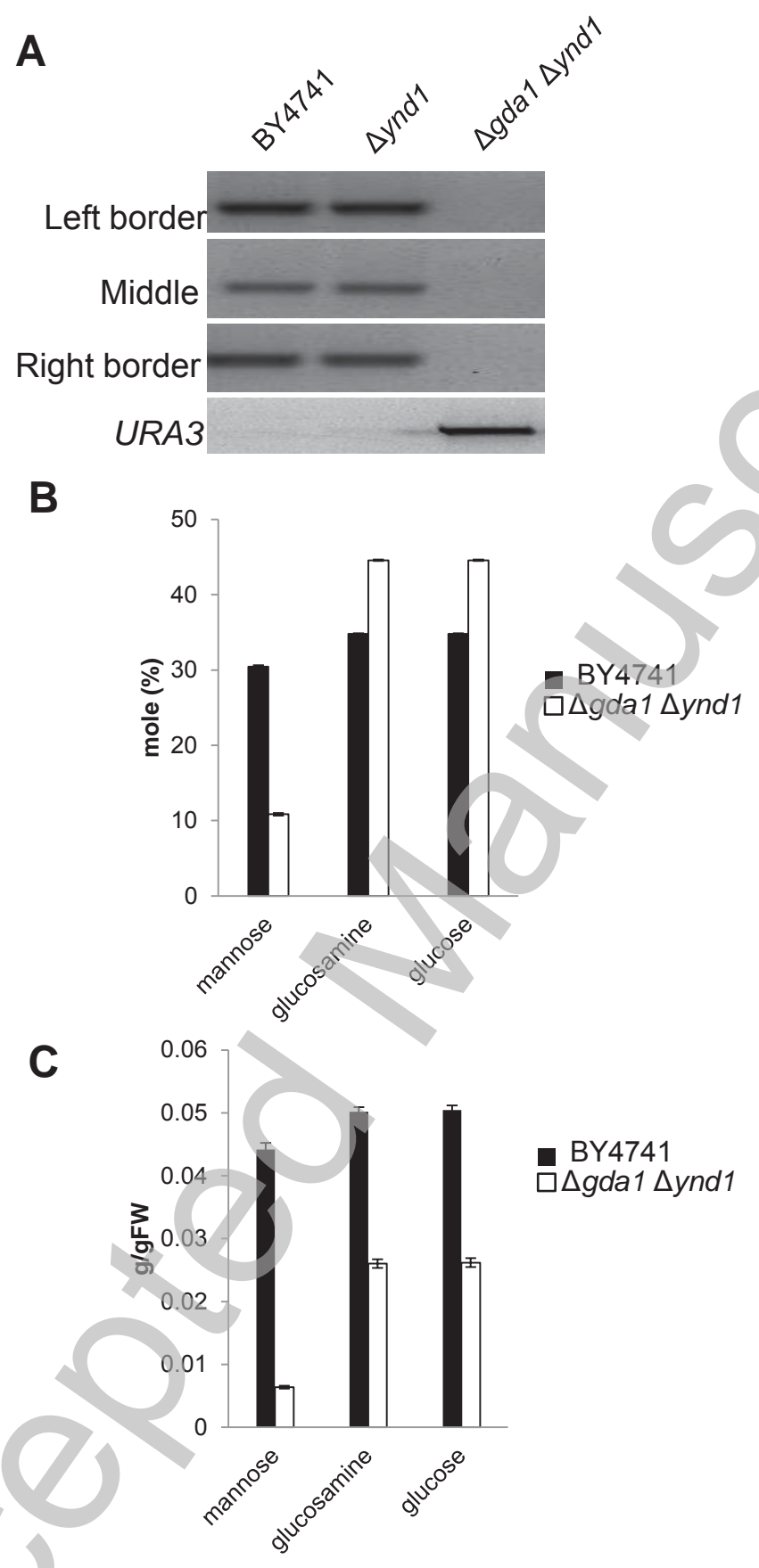

833

834 Figure 2.

835 
A

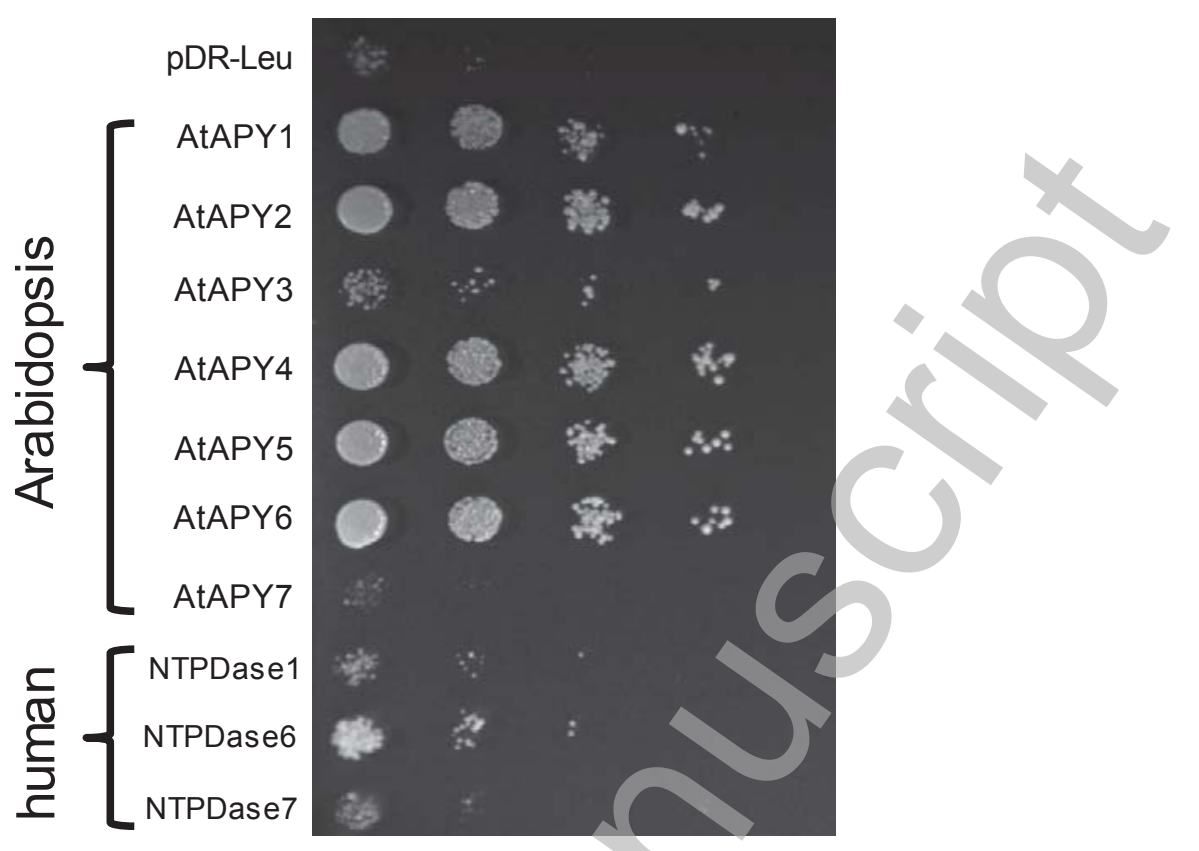

B

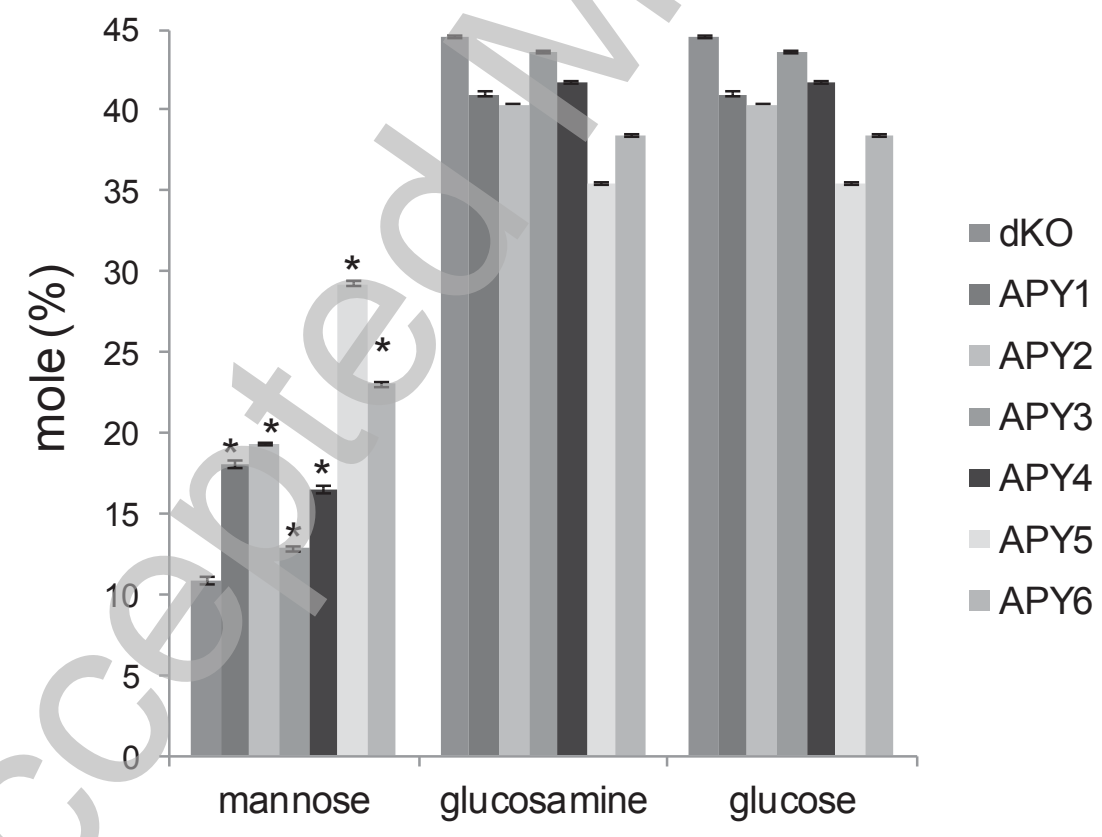

837 Figure 3.

838

839 

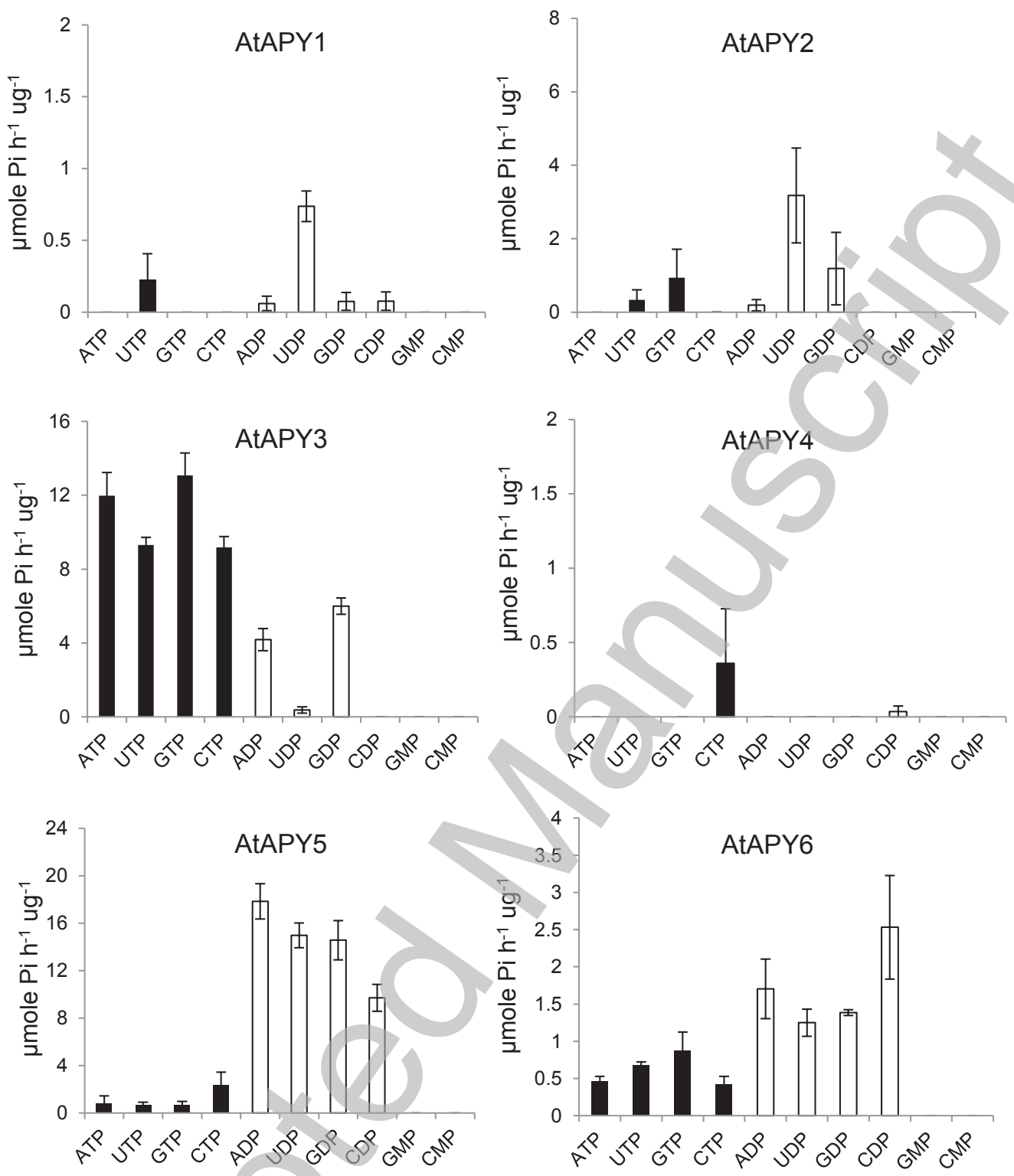

840

Figure 4.

842

843 


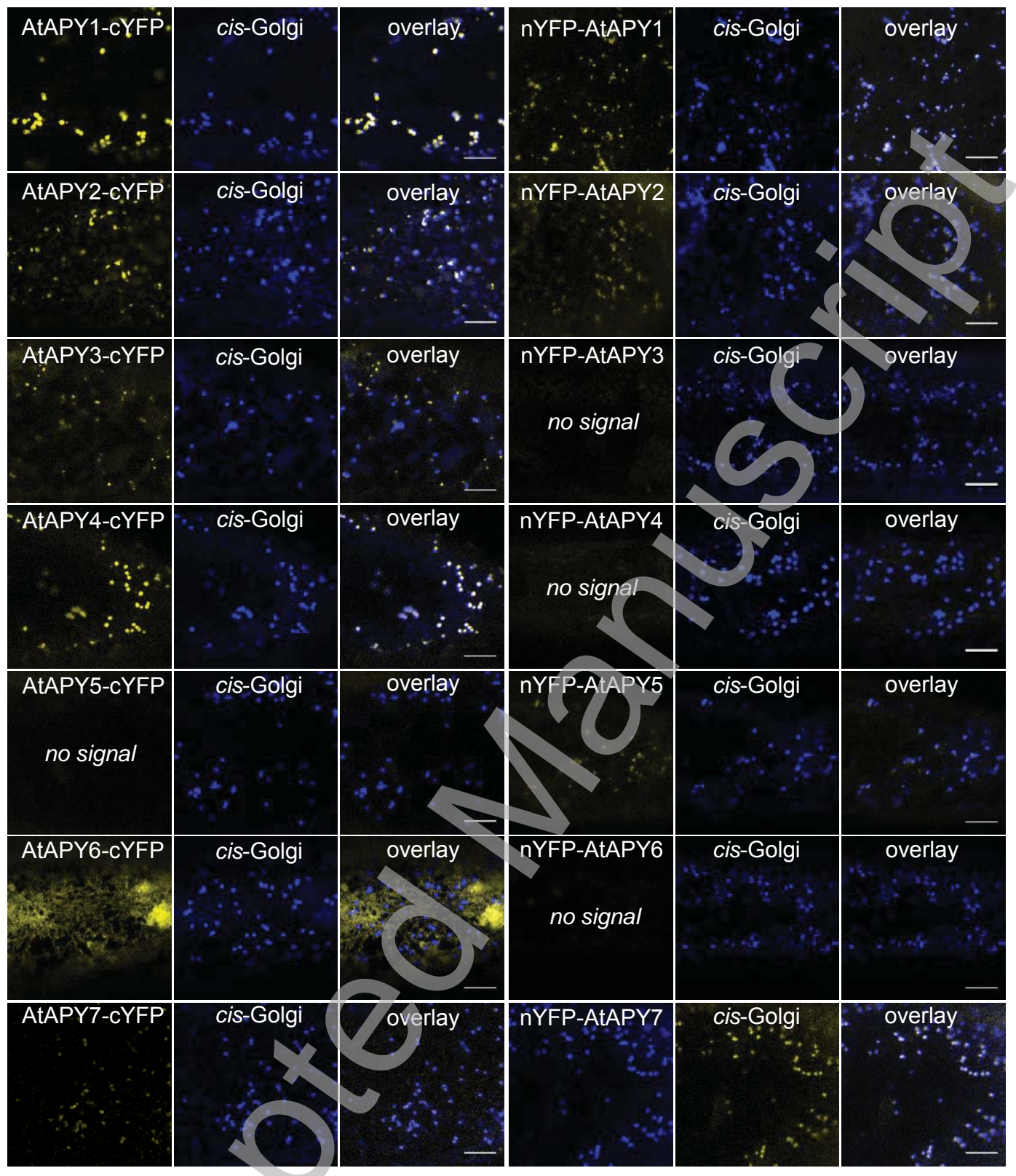

Figure 5. 


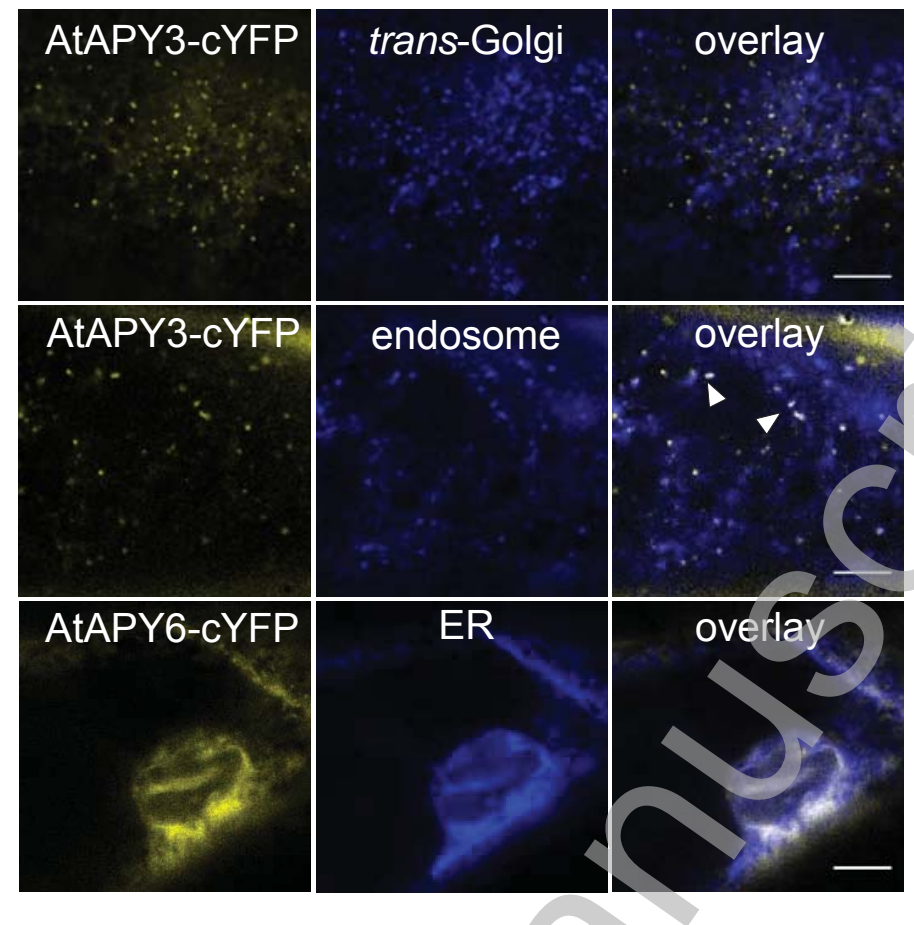

851 Figure 6. 


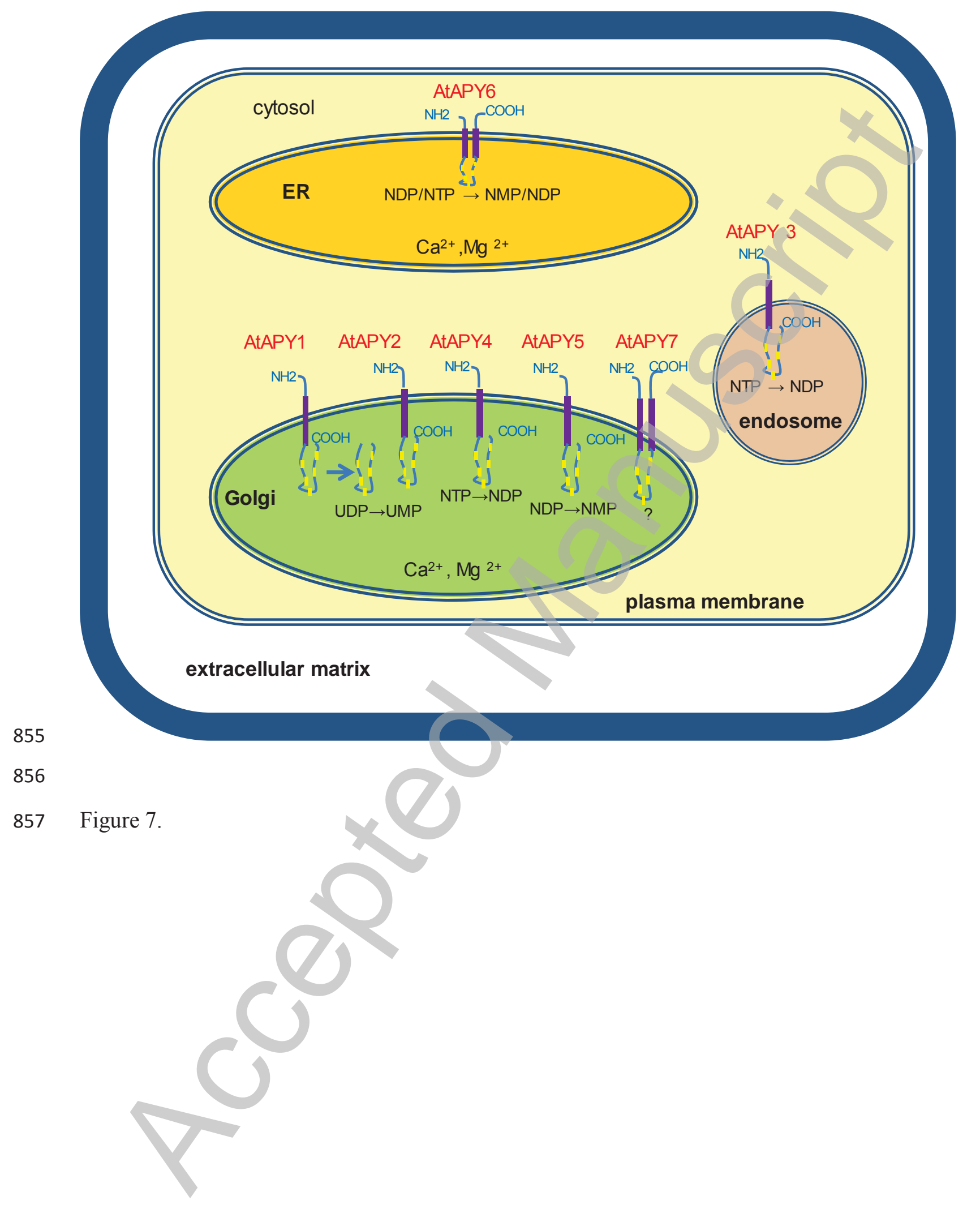




\section{University Library}

\section{- M M N E R VA A gateway to Melbourne's research publications}

Minerva Access is the Institutional Repository of The University of Melbourne

\section{Author/s:}

Chiu, T-Y;Lao, J;Manalansan, B;Loque, D;Roux, SJ;Heazlewood, JL

Title:

Biochemical characterization of Arabidopsis APYRASE family reveals their roles in regulating endomembrane NDP/NMP homoeostasis

\section{Date:}

2015-11-15

\section{Citation:}

Chiu, T. -Y., Lao, J., Manalansan, B., Loque, D., Roux, S. J. \& Heazlewood, J. L. (2015). Biochemical characterization of Arabidopsis APYRASE family reveals their roles in regulating endomembrane NDP/NMP homoeostasis. BIOCHEMICAL JOURNAL, 472 (1), pp.43-54. https://doi.org/10.1042/BJ20150235.

Persistent Link:

http://hdl.handle.net/11343/116464 